\title{
IDENTIFYING VECTOR CALCULUS TOPICS FOR INNOVATIVE TEACHING VIA COMPUTATIONAL TOOLS
}

\author{
N. Lohgheswary \\ Faculty of Engineering and Built Environment, SEGi University, \\ lohgheswarynagarethinam@gmail.com \\ Z. M. Nopiah \\ Faculty of Engineering and Built Environment, Universiti Kebangsaan Malaysia
}

A. A. Aziz

Faculty of Computing and IT, King Abdulaziz University

E. Zakaria

Faculty of Education, Universiti Kebangsaan Malaysia

\begin{abstract}
Understanding abstract engineering mathematics concepts for engineering students especially in Vector Calculus has been a nationwide issue. The effect of this situation is the poor performance of engineering students. This study compares Universiti Kebangsaan Malaysia's (UKM) weekly syllabus of Vector Calculus with some other 59 universities across the world to find the common topics in Vector Calculus. These topics will be included in lab sessions, an innovation way of teaching Vector Calculus. These topics will be included in lab sessions, an innovative way of teaching Vector Calculus. Teaching Vector Calculus using computational tools has been shown positive result by many recent studies. Basic complex number, vector functions, partial derivatives, line integrals, double integral, triple integral, Green's theorem, Stokes' theorem are the common topics in Vector Calculus which are suggested for lab session will be conducted for two hours and this will add to a total of eight weeks of laboratory sessions. In future it is suggested that laboratory sessions should be a part of Vector Calculus syllabus. The curriculum of Vector Calculus subject needs to be reviewed. This innovative teaching method helps to visualize graphs and understand difficult concept in Vector Calculus tremendously. In addition, tedious calculation can be computed easily using the computational method.
\end{abstract}

Keywords: Engineering mathematics, Vector calculus, Weekly syllabus, Computational tools, Innovative teaching

\section{Introduction}

All the first year engineering students will be studying Vector Calculus subject in their first year of engineering courses. A concrete understanding of this subject is needed as it will be applied in other engineering courses. Students need to ensure that they understand the underlying concept of Vector Calculus subject very well.

The decline in learning Engineering Mathematics is because of the students' poor visualization in the applications of mathematical concepts in real-life engineering problems. According to Adair and Desmond (2014), although lecturers include application examples in their lectures, students still fail to see "real" engineering problems.

Recently, many researchers have integrated teaching engineering mathematics using computational tools. Tokes et al. (2005) conducted MATLAB classes at the University of Queensland in 2002. Precalculus, Calculus of one variable, Calculus of many variables, Linear Algebra and Ordinary Differential Equations are among the courses conducted using MATLAB. Each module includes mathematical concepts, examples and exercises. Students started by running simple codes to familiarize themselves with MATLAB. Next they used GUTS from MATLAB to visualize difficult mathematical concepts. Finally the students developed their own coding in MATLAB. Students' feedback was very positive in incorporating MATLAB in teaching and learning. 
Although lecturers spend more time to illustrating the underlying concept of three-dimensional Calculus, students tend to find it difficult to understand. To overcome this problem, Cook (2006) used the Maple graphing tool to teach-dimensional Calculus subject. Instead of classroom teaching, class projects were conducted for the functions of two variables, Lagrange multipliers, line integrals and plotting secant vectors. The projects aim to help students to understand the mathematical concepts through visualization.

Synder (2006) used Maple to improve the depth of students' conceptual understanding of Calculus. In addition Maple was used as a problem solving tool. Before releasing the assignments, two sessions of learning Maple were installed in all computer labs for easy access for students. Individual tutorials assisted students in learning Maple. Five individual assignments and one group assignment aim to make students learn Calculus via Maple software. This study suggests that Maple should be integrated in the Calculus course and should be used regularly in classes.

Kovacheva (2007) applied Maple in Calculus, Linear Algebra, Ordinary Differential Equations, Numerical Methods and Statistics. Maple was incorporated in laboratory exercises. Students engaged themselves in problem-solving via Maple. Maple enhanced the depth of the comprehension of a subject and increased students' motivation.

Suanmali (2008) engaged Maple as a multimedia tool in classrooms. Engineering students who enrolled for Calculus had the opportunity to learn Maple for their assignments. Complex theory in Calculus for instance, Riemann Sum for $\mathrm{y}=\mathrm{f}(\mathrm{x})$ on the interval [a,b] was learned by Maple via visualization. Students were excited to learn Maple and Maple engaged students in learning.

Dikovic' (2009) explored Differential Calculus using GeoGebra. Firstly lectures were conducted in the traditional class method. Later, an experimental group which consisted of 31 students worked in a computer laboratory where the lecturer acted as a coordinator. Group work, individual research and investigations were among the tasks assigned to students. A pre-test was conducted at the end of the session. Post-test score was higher than the pre-test score. This proves that technological tool is a powerful tool for simulation and visualization of important topics of Differential Calculus.

Godarzi (2009) investigated the procedural and conceptual knowledge in teaching-learning of double integral using Maple 12. 44 students were chosen randomly and divided into control and experimental groups. Six sessions with one and half hours for each session were allocated for both groups. A pre-post test was administered to both groups. Students' pre-test scores showed no differences. Yet the post-test concluded that the experimental group had a better conceptual and procedural knowledge compared to the control group. Students agreed that Maple 12 was helpful in visualizing the basic concepts of multivariable Calculus.

Noinang (2009) conducted Integral Calculus class using Maple worksheets and interactive Maplets. This helped the students in self-planned learning and self-assessment. Line integrals, surface integrals and volume integral were illustrated using Maplets. Maplet covers three main functions such as input functions to define a problem, also a graphic visualization function and output functions to demonstrate results. Students were able to check their answers using Maplets. Thus, the quick solution by Maplets and the enhanced visualization of Maplets reinforced students' conceptual understanding of Integral Calculus.

Sage, free open source software was used by Botana (2014) in teaching Advanced Calculus. A DVD was developed using Sage which contains 30 worksheets. Students were involved in class activity as they worked in a pairs. Students commented positively that they avoided wasting time in computations. They rather use time to understand other mathematical concepts.

Mathematica is integrated in Vector Calculus and Partial Differential Equation. Students' understanding increased and they had better understanding in facing real-life applications on engineering. Students showed more interest in studying engineering mathematics. A pre-test was conducted to 136 students and they were divided equally into control and experimental groups. Six laboratories were conducted for the experimental group while control group had six sessions of extra tutorial. Later, both groups had post-tests. There is no difference in understanding simple concept of engineering mathematics.

All the above stated researchers found that including computational tools is a benefit for the students. The researchers have used different software to conduct lab. Maple, MATLAB, Sage and Mathematica are among the software used to conduct Vector Calculus lab.

This paper aims to review UKM's Vector Calculus syllabus with other public universities' and some world top universities' syllabi to identify the important topics in 14-week curriculum.

Methodology 
In UKM, Vector Calculus is a common subject for all engineering departments and is taken in the first semester of study. The duration of this subject is 14 weeks and it is a 4 credit hour subject. The weekly syllabus for Vector Calculus subject in UKM is given in Table 1.

Table 1: The weekly syllabus of Vector Calculus in UKM

\begin{tabular}{c|c} 
Week & Syllabus \\
\hline 1 & Understanding basic complex and hyperbolic function. \\
3 & Vector functions. \\
4 & Motion on a curve. Curvature and components of acceleration. \\
5 & Partial derivatives. Directional derivatives. \\
6 & Tangent planes and normal lines. Divergence and curl. \\
7 & Line integrals. Independence of path. \\
8 & Double integrals. Double integrals in polar coordinates. \\
9 & Green's theorem. Surface integrals. \\
10 & Stoke's theorem. \\
11 & Triple integrals. \\
12 & Sets in the complex plane. Functions of a complex variable. Cauchy-Riemann equations. \\
13 & Contour integrals. Cauchy-Goursat theorem. \\
\hline 14 & Independence of path. Cauchy's integral formulas.
\end{tabular}

The UKM Vector Calculus syllabus is compared with that from 59 universities all over the world. The distribution of the universities is as follows. Twenty universities are selected from the United States. They are Massachusetts Institute of Technology, Stanford University, University of California, California Institute of Technology, Princeton University, Georgia Institute of Technology, Carnegie Mellon University, University of Texas at Austin, University of Michigan, Cornell University, University of Illinois at Urbana Champaign, Northwestern University, University of WisconsinMadison, Columbia University, University of Washington, University of Minnesota, Rice University, Purdue University, Ohio State University and Pennsylvania State University.

Twenty universities are selected from the United Kingdom. They are University of Cambridge, University of Oxford, Imperial College London, University of Manchester, University College London, University of Edinburgh, University of Nottingham, University of Bristol, University of Southampton, University of Leeds, University of Sheffield, University of Liverpool, The University of Warwick, University of Bath, University of Strathclyde, Cardiff University, New Castle University, Queen Mary University of London, University of Glassgow and University of Surrey.

Ten universities from Oceania are chosen for this comparative study. They are University of Melbourne, University of Queensland Australia, University of Sdyney, Monash University, University of New South Wales, University of Auckland, University of Otago, University of Canterbury, Victoria University of Wellington and Massey University.

In addition, 4 universities from Asia are selected for the comparative study. They are National University of Singapore, Nanyang Technological University, Hong Kong University of Science and Technology and The University of Hong Kong.

Five universities from Malaysia are chosen too for this study. They are Universiti Malaya, Universiti Teknologi Malaysia, Universiti Sains Malaysia, Universiti Putra Malaysia and Universiti Teknologi Mara. Firstly, each university website was browsed to get into the engineering faculty. Then all the engineering mathematics subjects were browsed to find the specific name of the subjects offered in the engineering department. Then each subject syllabus was explored thoroughly to find the 
match between UKM's Vector Calculus syllabus content. A table was prepared for each subject. If the other universities' syllabi have the same weekly topic as UKM's ' $x$ ' will be given.

Once the table was completed for the subject, the total ' $\mathrm{x}$ ' for 59 universities for week 1 was calculated. For example 'Understanding basic complex number and hyperbolic function' is taught by 30 universities out of 59 universities. Hence, the percentage for that topic is calculated in the following way.

$$
\frac{30}{59} \times 100=51 \%
$$

In the same way, the other 13 weekly topics' percentages were calculated.

\section{Results and Discussion}

Table 2 and Table 3 show the match between the UKM's and world top universities' Vector Calculus syllabus. Table 4 shows the percentages for the weekly topic for Vector Calculus.

Table 4: Percentage of weekly topic for Vector Calculus

\begin{tabular}{c|c|c}
\hline Week & Total & Percentage \\
\hline 1 & 30 & 51 \\
2 & 40 & 68 \\
3 & 13 & 22 \\
4 & 38 & 64 \\
5 & 28 & 47 \\
6 & 36 & 61 \\
7 & 45 & 76 \\
8 & 38 & 64 \\
9 & 32 & 54 \\
10 & 45 & 76 \\
11 & 24 & 41 \\
12 & 11 & 19 \\
\hline 13 & 6 & 10 \\
\hline 14 & 8 & 14 \\
\hline
\end{tabular}

From Table 4, a bar chart is drawn to represent the distribution percentages of the weekly topics of Vector Calculus. Fig. 1 shows the distribution of weekly topics for Vector Calculus.

Thus, the highest percentage of eight topics from Table 4 will be selected for lab sessions. Table 5 illustrates the suggested lab sessions for Vector Calculus subjects.

Table 5: Suggested lab sessions for Vector Calculus

\begin{tabular}{c|c} 
Week & Lab \\
\hline 1 & Understanding basic complex and hyperbolic function. \\
2 & Vector functions. \\
3 & Partial derivatives. Directional derivatives. \\
4 & Line integrals. Independence of path. \\
5 & Double integrals. Double integrals in polar coordinates. \\
6 & Green's theorem. Surface integrals. \\
7 & Stoke's theorem. \\
\hline 8 & Triple integrals.
\end{tabular}




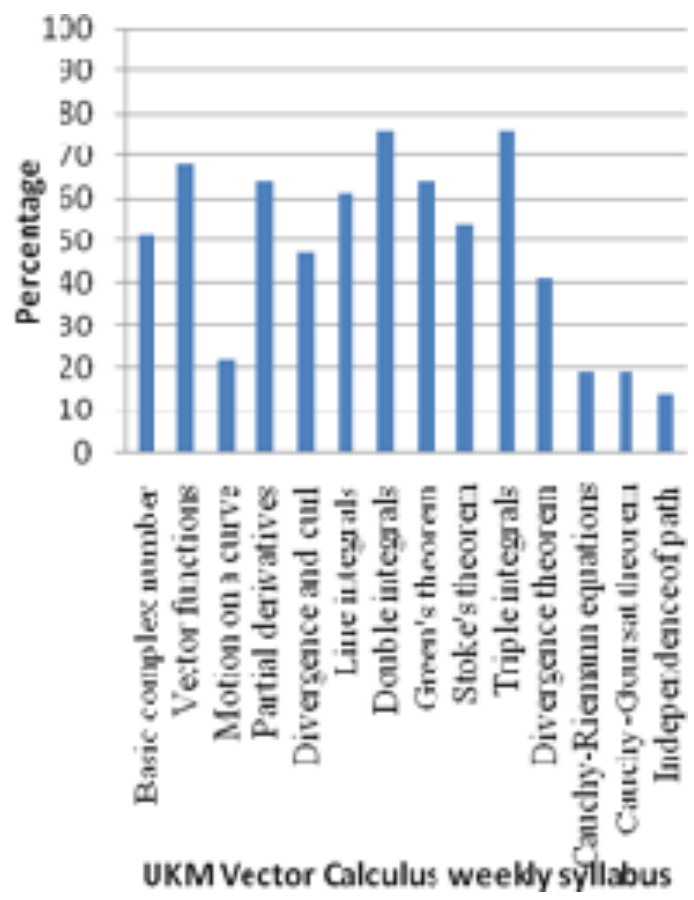

Fig. 1: Distribution of weekly topics for Vector Calculus

The eight highest percentage topics which is common between UKM and other universities are basic complex numbers, vector functions, partial derivatives, line integrals, double integrals, triple integrals, Greens' theorem, Stokes' theorem.

From Table 5, lab for week one encompasses the understanding of basic complex number and hyperbolic function. Second week's lab concentrates on vector functions. Cook (2006) used Maple graphing tool to plot secant vectors. Thus the graphing tool helped students to understand mathematical concepts via visualization.

Lab three covers partial derivatives and directional derivatives. Cook (2006) again used the Maple graphing tool to conduct class projects for the function of two or more variables.

Line integrals and the independence of path are suggested in the Vector Calculus topics for lab week four. Both Cook (2006) and Noinang (2009) used Maple in teaching line integrals. Cook (2006) used Maple graphing tool while Noinang 92009) used both the Maple worksheets and interactive Maple to conduct lab. Students will save a lot of time computing via Maple.

Lab for week five covers double integrals in polar coordinates. Godarzi (2009) taught double integral using Maple 12 and students completely agreed that Maple 12 helped in visualizing basic concepts of Calculus.

Next, Green's theorem and surface integrals are the suggested lab topics for week six. Noinang (2009) who used Maple in teaching surface integrals concluded that the experimental group had better conceptual and procedural knowledge compared to the control group.

Lab for week seven is Stokes' theorem. Adair and Desmond (2014) who conducted the lesson for Stokes' theorem using Mathematica agreed that Mathematica helped student in understanding tough concepts in Engineering Mathematics. 
The last lab is triple integrals. Noinang (2009) who integrated Maple worksheets and interactive Maplets in studying volume integral agreed that Maple software reinforced students' conceptual understanding in learning.

All the researchers illustrated the benefit of including computational tools and generally positive feedback obtained from students for this method of teaching. It is suggested that UKM's Vector Calculus curriculum needs to be readjusted to include the lab session as an innovative teaching method. UKM's engineering students will have a better conceptual understanding on Vector Calculus.

\section{Conclusion}

Difficulties in learning Vector Calculus subjects prove to be an issue faced by engineering students. The abstract concepts which were taught in a traditional way did not help the students to fully understand the content. Thus, an innovative learning method via computational tool is proposed in this study. Eight weeks of laboratory topics were chosen for this new method of teaching. The Vector Calculus syllabus of UKM is compared with 59 local and international universities to find out the common topics for the lab sessions. Other recent researchers have provided positive feedback regarding the inclusion of lab sessions in engineering mathematics courses. Incorporating computing tool in the teaching of Vector Calculus will therefore, help UKM engineering students to understand the abstract mathematical concepts in Vector Calculus. Thus, it will improve students' performance in Vector Calculus and other engineering mathematics subjects.

\section{Acknowledgment}

The authors wish to express gratitude towards SEGi University and Universiti Kebangsaan Malaysia for supporting the research.

\section{References}

Adair D., and Jaeger M. (2014). Making Engineering Mathematics More Relevant Using a Computer Algebra System. International Journal of Engineering Education, 30(1): 199-209.

Botana F., Abanades M. A., and Escribano I. (2014). Using Free Open Source Software To Teach Mathematics. Computer Applications in Engineering Education, 22(4): 728-735.

Cook D. (2006). Maple Graphing Tool for Calculus III. Mathematics and Computer Education, 40(1): 36-41.

Dikovic' L. (2009). Application GeoGebra into Teaching Some Topics of Mathematics at the College Level, 6(2): 191-203.

In proceedings Godarzi S. Q., Aminifar E., and Bakhshalizadeh S. (2009). The Impact of Using Computer Algebraic System in Teaching and Learning of "Double Integral".

Kocacheva T. (2007). Use of Maple System in Math Tuition at Universities. International Journal Information Technologies and Knowledge, 1: 363-368.

Noinang S., Wiwatanapataphee B., and Wu Y. H. (2009). Teaching-Learning Tool for Integral Calculus. For Far East Journal of Mathematical Education, 3(3): 203-211.

Suanmali S. (2008). Maple in Mathematics. Fifth International Conference on Information Technology: New Generation, 528-533.

Synder K. (2006). Student's Emerging Understanding of the Command-Driven Computer Algebra System Maple in a Semester Calculus Course. Doctoral Dissertation. Wayne State University.

Tonkes E. J., Loch B. L. and Stace A. W. (2005). An Innovation Learning Model for Computation in First Year Mathematics Education in Science and Technology, 36(7): 751-758.

Table 2: Matching of UKM and other universities Vector Calculus syllabus 


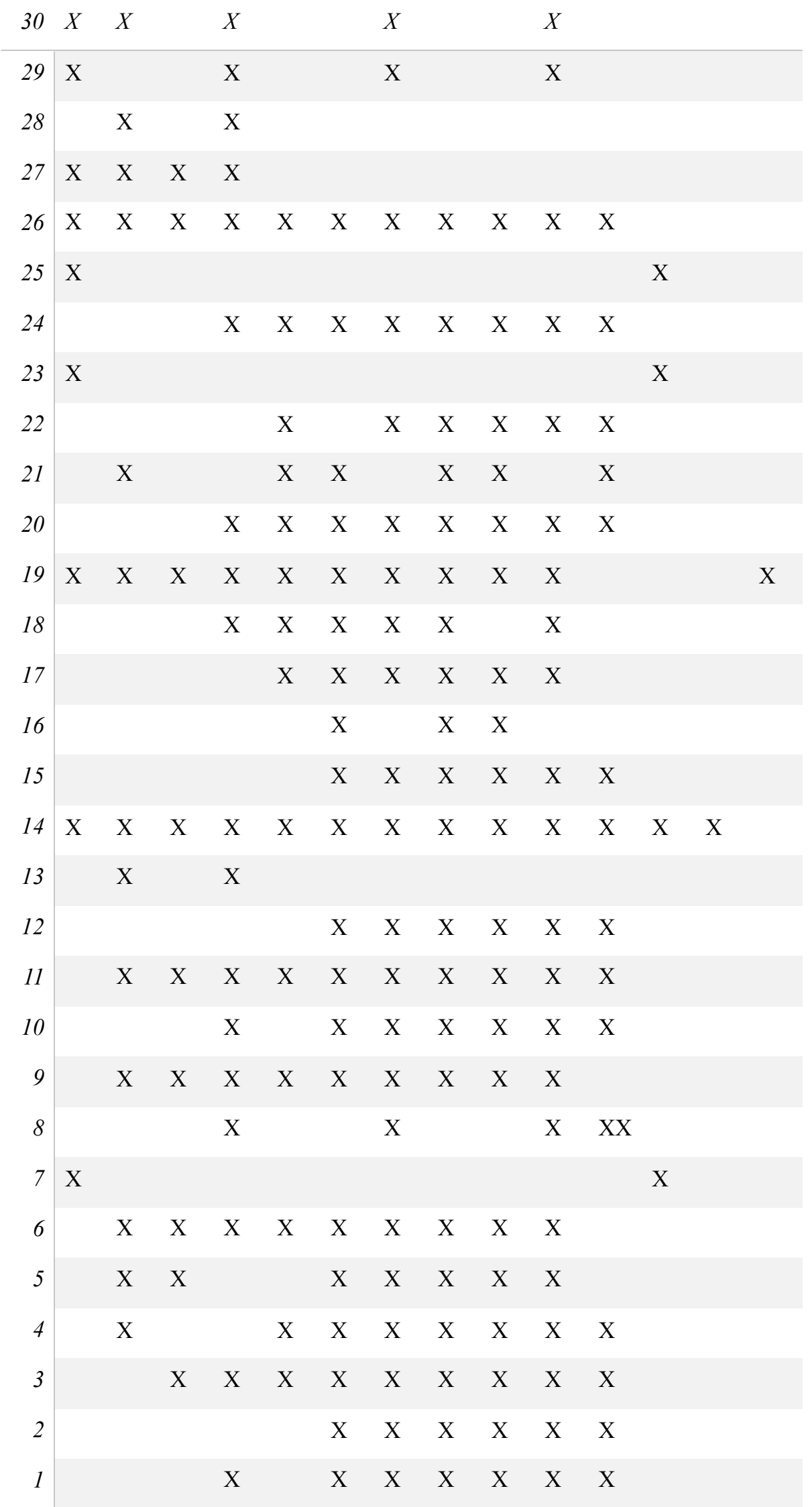




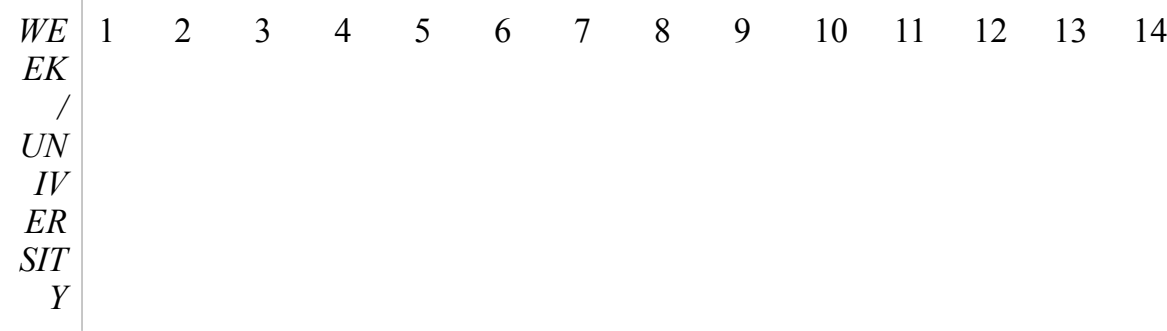

Table 3: Matching of UKM and other universities Vector Calculus syllabus

\begin{tabular}{|c|c|c|c|c|c|c|c|c|c|c|c|c|c|c|}
\hline 59 & $X$ & $X$ & & $X$ & & & $X$ & & & $X$ & & & & \\
\hline 58 & $X$ & $X$ & & & & & & $X$ & $X$ & $X$ & & $X$ & $X$ & \\
\hline 57 & $X$ & $X$ & & $X$ & & $X$ & $X$ & $X$ & $X$ & $X$ & & $X$ & & $X$ \\
\hline 56 & & $X$ & & $X$ & $X$ & $X$ & $X$ & $X$ & $X$ & $X$ & $X$ & & & \\
\hline 55 & $X$ & & & $X$ & & & & & & & & & & \\
\hline 54 & $\mathrm{X}$ & $X$ & $\mathrm{X}$ & $X$ & & $X$ & $X$ & $X$ & $X$ & $X$ & & & & \\
\hline 53 & & $X$ & $X$ & $X$ & $X$ & $X$ & $X$ & $X$ & & $X$ & & & & \\
\hline 52 & $X$ & $X$ & & $X$ & & & $X$ & & & $X$ & & $X$ & & $X$ \\
\hline 51 & $X$ & $X$ & & $X$ & & & $X$ & & & $\mathrm{X}$ & & $X$ & & $X$ \\
\hline 50 & $X$ & $X$ & & & & & & & & & & & & \\
\hline 49 & $\mathrm{X}$ & $X$ & & $X$ & $X$ & $X$ & $X$ & $X$ & $X$ & $X$ & $X$ & & & \\
\hline 48 & & $X$ & & & & $X$ & $X$ & & & $X$ & & & & \\
\hline 47 & $\mathrm{X}$ & $X$ & & $\mathrm{X}$ & $X$ & $X$ & $X$ & $X$ & & $X$ & & $X$ & $X$ & $X$ \\
\hline 46 & $X$ & & & & & & & & & & & $X$ & $X$ & $X$ \\
\hline 45 & $X$ & $X$ & $X$ & $X$ & $X$ & $X$ & $X$ & $X$ & $X$ & $X$ & $X$ & & $X$ & $X$ \\
\hline 44 & $X$ & $X$ & $X$ & $X$ & $X$ & $X$ & $X$ & $X$ & $X$ & $X$ & $X$ & $X$ & & X \\
\hline 43 & & $X$ & & & $X$ & $X$ & $X$ & $X$ & $X$ & $X$ & $X$ & & & \\
\hline 42 & & $X$ & & $X$ & & $X$ & & & & & & & & \\
\hline 41 & $X$ & $X$ & & $X$ & & & & $X$ & $X$ & $X$ & $X$ & & & \\
\hline 40 & $X$ & $X$ & & $X$ & & & $X$ & & & $X$ & & & & \\
\hline 39 & $X$ & $X$ & & $X$ & & $X$ & $X$ & & & & & & & \\
\hline 38 & & $X$ & & & & & $X$ & $X$ & & $X$ & & & & \\
\hline 37 & $X$ & $X$ & & & $X$ & & $X$ & $X$ & & $X$ & $X$ & & & \\
\hline 36 & $X$ & $X$ & & $X$ & $X$ & $X$ & $X$ & $X$ & & $X$ & & & & \\
\hline 35 & $X$ & $X$ & & & $X$ & & $X$ & & & & & & & \\
\hline
\end{tabular}


The Turkish Online Journal of Design, Art and Communication - TOJDAC

ISSN: 2146-5193, September 2018 Special Edition, p.1121-1129

\begin{tabular}{|c|c|c|c|c|c|c|c|c|c|c|c|c|c|c|}
\hline 34 & & $X$ & & $X$ & $X$ & $X$ & $X$ & X & $X$ & $X$ & & & & \\
\hline 33 & & & & X & X & & $X$ & & & $X$ & & & & \\
\hline 32 & X & $X$ & & & X & $X$ & $X$ & X & X & X & & & & \\
\hline 31 & X & $X$ & & X & $X$ & $X$ & $X$ & $X$ & $X$ & $X$ & $X$ & & $X$ & \\
\hline $\begin{array}{r}W E \\
E K \\
/ \\
U N \\
I V \\
E R \\
S I T \\
Y\end{array}$ & 1 & 2 & 3 & 4 & 5 & 6 & 7 & 8 & 9 & 10 & 11 & 12 & 13 & 14 \\
\hline
\end{tabular}

